\title{
Resource allocation for virtual reality content sharing based on 5G D2D multicast communication
}

\author{
Yang Yang ${ }^{1}$, Lei Feng ${ }^{1 *}$, Cheng Zhang ${ }^{2}$, Qinghai $\mathrm{Ou}^{3}$ and Wenjing $\mathrm{Li}^{1}$
}

\begin{abstract}
As the development of wireless virtual reality (VR), a great disparity exists among the huge content transmission, rigorous QoS guarantees and the limited bandwidth of cellular networks. With the increasing of cache capacity on user devices, direct content sharing between user devices is a promising solution to solve this problem. To meet the quality of service (QoS) requirement of wireless VR transmission, this paper proposes a content sharing scheme based on 5 G device-to-device (D2D) Multicast Communication. In this way, some adjacent VR users can form multicasting clustering to share VR content by working on D2D communication mode. The VR D2D multicasting clusters reuse the uplink channel resource of ordinary VR cellular users in the same cell. The basic prerequisite is that the degrading on QoS of these ordinary VR cellular users can be affordable. We design a two-step scheme to solve this radio resource allocation problem for VR content sharing. Firstly, we find out the optimal transmitting power for each VR user devices by geometric proximity, which metrics are affected by wireless VR throughput, tracking accuracy and delay. In the second step, we transform the channel allocation problem into a bipartite graph matching problem based on the transmitting power metrics, which is optimally solved by the Hungarian algorithm. The simulation results show that the VR content sharing based on 5G D2D communication technology can achieve larger system throughput gain and lower transmission delay. Compared with heuristic scheme and stochastic scheme, the proposed scheme can increase the throughput of the overall network by about $50 \%$ and $12 \%$, respectively.
\end{abstract}

Keywords: Virtual reality, D2D, Multicast communication, Content sharing, Resources allocation, Power control

\section{Introduction}

Virtual reality (VR), known as "the most promising application" [1]. The users can have an immersive experience in the virtual environment and interact with it through some head-mounted devices [2]. Good experience of VR service means low latency and high resolution when transmitting lots of content. While in its infancy, quality of VR services is guaranteed by solid and reliable fiber communications [3], which have poor quality of service (QoS). As the rapid development of $5 \mathrm{G}$ cellular networks, wireless VR is increasingly researched and achieved and the quality of

\footnotetext{
* Correspondence: fenglei@bupt.edu.cn

'Institute of Network Technology, Beijing University of Posts and

Telecommunications, Xitucheng Road, Beijing 100876, China

Full list of author information is available at the end of the article
}

service is guaranteed as well. Different from optical communications which are relatively fixed, VR services are led to be truly "anywhere" by $5 \mathrm{G}$ wireless transmission.

However, the field of wireless VR faces some challenges that include vertigo and limited bandwidth. According to many practical application measurements, some users will feel vertigo in VR's head-mounted devices if the delay exceeds $10 \mathrm{~ms}$ [4]. At present, this delay requirement of VR cannot be guaranteed even with $5 \mathrm{G}$ cellular networks. The reasons are the transmission latency occurred in the long-distance core network and the processing delay of the VR computing. In addition, it can be foreseen that VR users will grow dramatically. The delivery of the massive common VR content of high clarity to the densely distributed users may bring a heavy burden to the $5 \mathrm{G}$ base

\section{Springer Open}

(๑) The Author(s). 2020 Open Access This article is licensed under a Creative Commons Attribution 4.0 International License, which permits use, sharing, adaptation, distribution and reproduction in any medium or format, as long as you give appropriate credit to the original author(s) and the source, provide a link to the Creative Commons licence, and indicate if changes were made. The images or other third party material in this article are included in the article's Creative Commons licence, unless indicated otherwise in a credit line to the material. If material is not included in the article's Creative Commons licence and your intended use is not permitted by statutory regulation or exceeds the permitted use, you will need to obtain permission directly from the copyright holder. To view a copy of this licence, visit http://creativecommons.org/licenses/by/4.0/. 
station since of the limited bandwidth. The burden to the 5G base station since of the limited bandwidth. Therefore, how to reduce the delay of content delivery and achieve content sharing becomes a key issue for the development of wireless VR services.

D2D which is one of the important $5 \mathrm{G}$ technologies can reuse the uplink channel resources of cellular users so that adjacent devices can communicate directly with each other without access to base stations [5]. Combining with multicasting technology in further, D2D communication can simultaneously send the content of common interest to multiple neighboring users. When this combination is applied in the field of wireless VR transmission, it cannot only offload the traffic of the cellular networks and reduce latency, but also promote clarity of VR content by a lot of large improvement to the throughput [6]. Therefore, we achieve wireless VR content sharing based on D2D multicasting technology and work on its resource allocation scheme in this paper. Our goal is to maximize the improvements in the downlink throughput of VR users in D2D multicasting clusters if the quality of experience (QoE) degradation can be affordable for some other VR devices whose uplink channels resources are multiplexed by theses D2D multicasting clusters.

In this paper, we assume that some VR devices with D2D capabilities are deployed within a $5 \mathrm{G}$ cell and intend to implement real immersive content sharing with each other. There are two kinds of VR communication modes in this scenario: the VR users who have the same VR content sharing intent form the D2D multicasting clusters and other VR users communicate directly with the base station. The D2D multicasting clusters reuse the uplink channel resources which are occupied by non-clustered VR users to send tracking information to the base station. After that, the problem of resource allocation considering the connectivity of D2D multicast cluster and the quality of service of virtual reality users is studied. Based on the optimal power and channel allocation, the D2D multicasting cluster throughput for VR content sharing is maximized. Our contributions are summarized as follows:

1. D2D multicasting is innovatively utilized to improve the clarity and rate performance of VR content sharing. In this case, the devices in the same cluster transmit information in multicasting mode to realizes VR video content sharing, which has more reasonable resource utilization and better QoS, like latency and clarity.

2. A novel resource allocation optimized problem is proposed to maximize the throughput of VR video content sharing constrained by the acceptable degradation on the uplink performance of the resource-reused VR users. In this model, several key VR performance metrics are taken into account including tracking accuracy, uplink transmission latency, and tracking processing delay.

3. Aiming at the above optimization problem, a twostep algorithm is proposed. Firstly, all the optimally feasible power assignments are calculated by the linear programming method. Then, we obtain the best channel allocation by the maximum weight bipartite graph matching algorithm based on these feasible power assignments. The numerical simulations verify the proposed solution can effectively enhance the clarity of VR content transmission.

The remainder of this paper is organized as follows. We give a review of related work in Section 2. In Section 3 , we introduce the system model architecture. Section 4 formulates system optimization problems. Section 5 puts forward a two-step scheme to solve the problem. Section 6 gives the numerical simulations and analysis, and Section 7 gives some conclusions.

\section{Related work}

\subsection{Transmission of VR content}

Academia, industry, and other fields are unprecedentedly interested in VR and devoting a lot of work to it.

In order to guide users to move in VR immersion environment, [7] proposes an enhanced learning algorithm to facilitate users' interaction with a virtual environment. Reference [8] develops a transfer learning algorithm which is according to the liquid state machine to optimize the VR users' successful transmission probability. Reference [9] proposes a virtual reality live system to ensure the quality of service. In the work of [10], a concurrence multipath transmission-distortion aware (CMT-DA) scheme is proposed. The scheme consists of three stages: by analyzing the data distribution of multiple communication paths, quality of video can be improved. In [11], the quality of high-frame video transmission was improved by modeling. In Reference [12], a joint source-channel coding scheme according to forward error correction (FEC) is proposed for real-time VR applications. However, in the works of [10-12], only the transmission of video content is considered, and the generation of virtual video content and its relationship with tracking are not considered. In the work of [13], it proposed a virtual reality model of multi-attribute utility theory, which based on virtual reality indicators such as latency and tracking accuracy. Most of the existing VR work focuses on VR systems deployed on wired networks. Hence, the challenges faced by VR in wireless cellular networks are not applicable. Most of the existing researches on VR in wireless networks are focused on VR video quality and content transmission, without considering VR video content sharing. 


\subsection{D2D transmission technology}

Immersive technologies require tremendous improvements in bandwidth, latency, and reliability. Network congestion can be alleviated by using short-range communication technologies such as D2D and edge proximity services between VR users. Its purpose is to extract and share context information of VR users in view and camera transmission [14]. The reason why D2D technology is chosen is that short-range communication technologies such as WiFi and Zigbee are not flexible and need complex users configuration, and coordination before actual transmission [15]. In addition, these methods are mainly used for local direct sharing, which is not helpful for local-request sharing [16]. Local-direct sharing means that the content sharer has the requested content and can share it with other users directly. Localrequest sharing means that the content sharer does not contain the requested content and needs to send a request to the nearby base station to obtain the content before sharing it with other users. In addition to the above reasons, D2D technology has advantages in content sharing in the existing work. In Reference [17], taking the social relationship and trust between users as indicators, when they reach certain thresholds, D2D links are established within the group. And the users with the greatest trust are used as cluster heads to enhance the QoE of users. Reference [18] designed an effective resource allocation strategy to ensure the fairness of resource allocation among D2D multicast clusters. Reference [19] proposed a distributed service video transmission scheme based on the QoE model and a channel allocation algorithm based on pervasive function. In order to adjust the video user experience, Reference [20] proposed an adaptive D2D video stream quality-of-service-aware channel resource matching scheme. Reference [21] analyzes how the combination of D2D and edge computing affects the QoE of video streaming clients and helps to reduce backhaul traffic. In Reference [22], a pre-caching algorithm based on QoE gain is proposed to realize the distribution of D2D to VR video.

In this paper, D2D technology is applied to VR video transmission, which reduces the load of the base station and the transmission latency of content distribution. While bringing the advantages of D2D technology into full play, it ensures the VR user experience and achieves the sharing and distribution of VR video content.

\section{System model}

\subsection{System description}

The system model is a typical cellular network single-cell scenario, including an SBS and multiple user devices in Fig. 1. Including VR users $\operatorname{VR}_{i}(i=1,2, \ldots I)$, Idenotes the number of VR users, and D2D clusters $D_{j}(j=1,2 \ldots)$ ), Jdenotes the number of D2D clusters. SBS collects channel information, users' requests. The power and spectrum resources of all users are managed by SBS. The channel bandwidth is $B$ and the noise is $\sigma^{2}$. The model parameters are shown in Table 1. VR users send tracking information to SBS through the uplink and SBS sends video to users through the down-link. D2D users reuse the uplink of VR users to transmit the required video to the users in the cluster. D2D clusters can reuse one or more VR user channel resources, while one VR user channel resources can only be reused by one D2D cluster. As shown in Fig. 1, cluster $D_{1}$ reuses both channel $F_{1}$ of $\mathrm{VR}_{1}$ user and channel $F_{2}$ of $\mathrm{VR}_{2}$ user. When the channel is reused, there will be interference between D2D clusters and VR users.

\subsection{System architecture}

\subsubsection{VR model}

Tracking VR tracking information includes location information and direction information, and SBS builds a virtual environment based on VR users tracking information. The tracking vector of each VR user $i$ is expressed as $W_{i}=\left[w_{i 1}\right.$, $\left.w_{i 2}, w_{i 3}, w_{i 4}, w_{i 5}, w_{i 6}\right]$, where $\left[w_{i 1}, w_{i 2}, w_{i 3}\right]$ represents the position coordinates of each VR user and $\left[w_{i 4}, w_{i 5}, w_{i 6}\right]$ represents the direction coordinates of each VR user. The channel of $\mathrm{VR}_{i}$ which is reused by $D_{j}$ will interfere with $\mathrm{VR}_{i}$ data transmission. The signal-to-noise ratio between $\mathrm{VR}_{i}$ and SBS uplink is expressed as $\mathrm{SINR}_{i, j}^{V R}$ :

$$
\operatorname{SINR}_{i, j}^{V R}=\frac{P_{i, j}^{V R} h_{i, B}}{\sigma^{2}+P_{i, j}^{D} h_{j, B}} .
$$

Where $P_{i, j}^{V R}$ is the transmission power of $\mathrm{VR}_{i}$ whose channel resources are reused by $D_{j} . h_{i, B}$ is the channel gain between $\mathrm{VR}_{i}$ and SBS. $P_{i, j}^{D}$ is the transmission power of $D_{j}$ reusing $\mathrm{VR}_{i}$ channel resources, and $h_{j, B}$ is the path loss

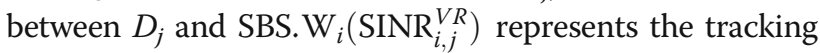
vector that $\mathrm{VR}_{i}$ transmits to $\mathrm{SBS}$, which is affected by the SINR of the wireless link.

For each VR user, the tracking accuracy $\mathrm{Z}_{i}^{V R}$ is expressed as follows :

$$
\mathrm{Z}_{i}^{V R}=1-\frac{\left\|W_{i}\left(\operatorname{SINR}_{i, j}^{V R}\right)-W_{i}^{R}\right\|}{\max \left\|W_{i}\left(\operatorname{SINR}_{i, j}^{V R}\right)-W_{i}^{R}\right\|} .
$$

Where $\left\|W_{i}\left(\operatorname{SINR}_{i, j}^{V R}\right)-W_{i}^{R}\right\|$ denotes the inaccuracy of VR tracking. Because of the influence of SINR, bit errors will occur in $W_{i}\left(\operatorname{SINR}_{i, j}^{V R}\right)$ transmission. Therefore, it has an impact on the

generation of video. $W_{i}^{R}$ means that when the user feels uncomfortable in the environment or is not satisfied with the video displayed, he sends a forced feedback to SBS.

Delay The latency mainly includes transmission latency (this paper only considers the uplink transmission 


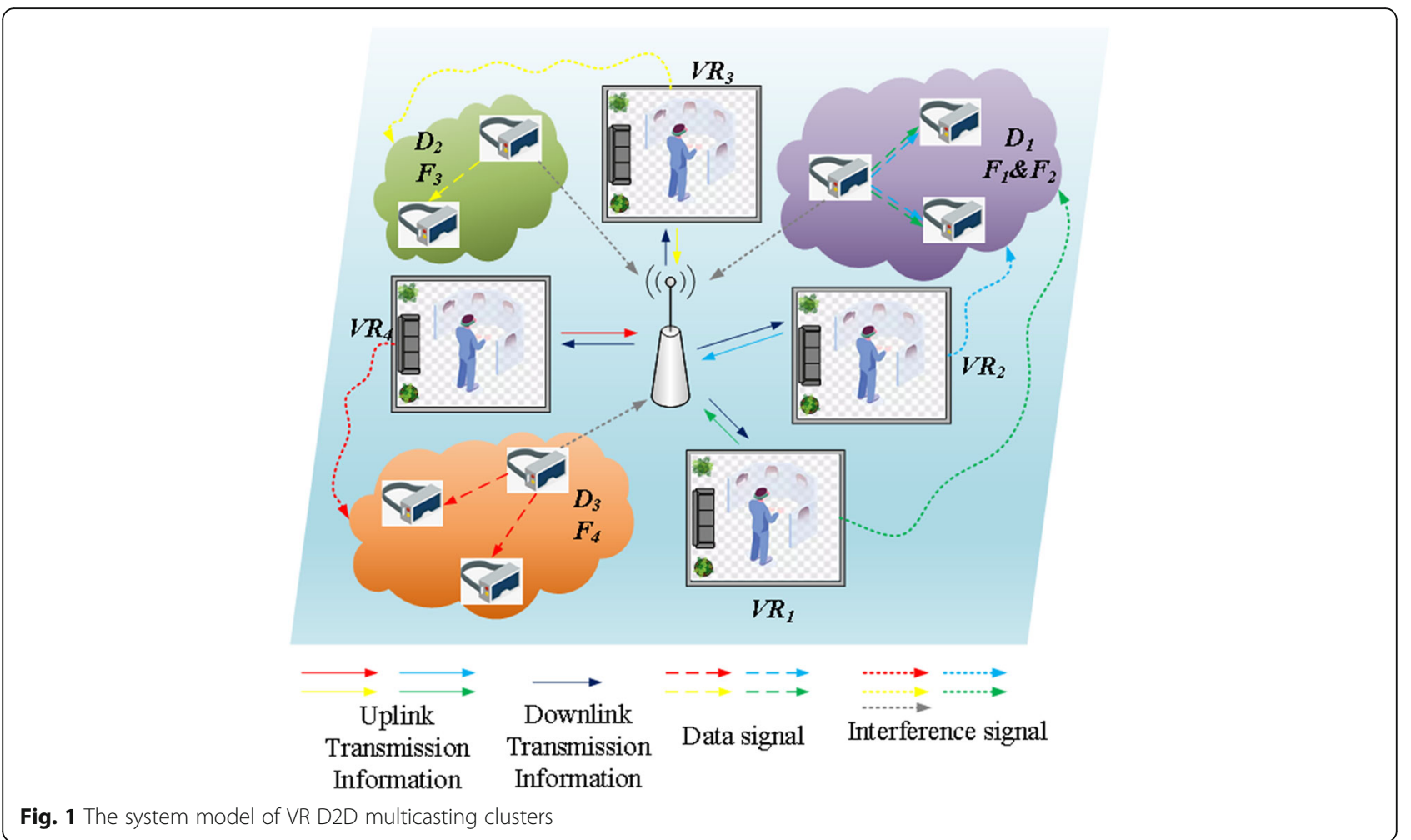

latency of VR users) and processing latency. The transmission latency for each VR user is as follows:

$$
V_{i}^{T}=\frac{A}{C_{i, j}^{V R}}
$$

Arepresents the size of the tracking vector sent to SBS by each VR user. $C_{i, j}^{V R}$ is the transmission rate of the VR user's uplink.

$$
C_{i, j}^{V R}=B \log _{2}\left(1+\operatorname{SINR}_{i, j}^{V R}\right),
$$

In processing delay, we mainly calculate the size of bits needed to be converted in video image generation. The vector $l\left(W_{i}\left(\operatorname{SINR}_{i, j}^{V R}\right)\right)$ represents the VR image generated

Table 1 Parameters of system

\begin{tabular}{ll}
\hline$I$ & The number of VR users \\
$\mathrm{B}$ & The number of D2D clusters \\
$\sigma^{2}$ & Channel bandwidth \\
$P_{i, j}^{V R}$ & Noise \\
$P_{i, j}^{D}$ & Transmission power of VR users \\
$V_{i}$ & Transmission power of D2D clusters \\
$Z_{i}^{V R}$ & The total delay of $\mathrm{VR}_{i}$ uplink transmission \\
\hline
\end{tabular}

by SBS according to the tracking vector $W_{i}\left(\operatorname{SINR}_{i, j}^{V R}\right)$. $l\left(W_{i}^{R}\right)$ represents the VR image generated by SBS according to the forced feedback tracking vector $W_{i}^{R}$. Since virtual reality system can predict future tracking information based on historical tracking information, $l\left(W_{i}\left(\operatorname{SINR}_{i, j}^{V R}\right)\right)$ has been generated before SBS receives $W_{i}^{R}$. The $l\left(W_{i}\left(\operatorname{SINR}_{i, j}^{V R}\right)\right)$ can be transferred into $l\left(W_{i}^{R}\right)$ using this time-saving method. Define $v\left(l\left(W_{i}\left(\operatorname{SINR}_{i, j}^{V R}\right)\right) l\left(W_{i}^{R}\right)\right)$ to denote the number of bits that need to be converted from VR image and they are determined by motion search algorithm [23]. Bit size is related to tracking accuracy, so processing delay is mainly affected by tracking accuracy. The processing delay is defined as follows :

$$
V_{i}^{P}\left(Z_{i}^{V R}\right)=\frac{v\left(l\left(W_{i}\left(\operatorname{SINR}_{i, j}^{V R}\right)\right) l\left(W_{i}^{R}\right)\right)}{M / N},
$$

where $0<=v\left(l\left(W_{i}\left(\operatorname{SINR}_{i, j}^{V R}\right)\right) l\left(W_{i}^{R}\right)\right)<=L$, the greater the difference between $l\left(W_{i}\left(\operatorname{SINR}_{i, j}^{V R}\right)\right)$ and $l\left(W_{i}^{R}\right)$, the more bits SBS needs to convert. $L$ represents the maximum number of bits allowed to be converted. $M / N$ is a computing resource for each VR user to process video. Therefore, the total latency for per user $\mathrm{VR}_{i}$ is 


$$
V_{i}=V_{i}^{P}\left(Z_{i}^{V R}\right)+V_{i}^{T}
$$

\subsubsection{D2D model}

In $D_{j}$, the D2D transmitter is represented as $d_{j, y}^{t}$, and the D2D receiver is represented as $d_{j, x}^{r} \cdot h_{j, d_{j, x}^{r}}$ denotes the channel gain between $d_{j, y}^{t}$ and $d_{j, x}^{r} . h_{i, d_{j, x}^{r}}$ denotes the channel gain between $\mathrm{VR}_{i}$ and $D_{j} . P_{i, j}^{V R}$ is the transmission power of $\mathrm{VR}_{i}$ whose channel resources are reused by $D_{j} . P_{i, j}^{D}$ is the transmission power of the D2D cluster $D_{j}$ which reuses $V R_{i}$ channel resources. Therefore, the SINR of $D_{j}$ is as follows:

$$
\begin{aligned}
\operatorname{SINR}_{i, j}^{D} & =\min _{x \in D_{j} \backslash\left\{y_{j}\right\}} \frac{P_{i, j}^{D} h_{j, d d_{j, x}^{r}}}{\sigma^{2}+P_{i, j}^{\mathrm{VR}} h_{i, d_{j, x}^{r}}} \\
& =\min \left(\frac{P_{i j}^{D} h_{j, d_{j, 1}^{r}}}{\sigma^{2}+P_{i, j}^{\mathrm{VR}} h_{i, d_{j, 1}^{r}}}, \ldots \frac{P_{i j}^{D} h_{j, d_{j, x}^{r}}}{\sigma^{2}+P_{i, j}^{\mathrm{VR}} h_{i, d_{j, x}^{r}}}\right) ;
\end{aligned}
$$

The throughput of $D_{j}$ reusing $V R_{i}$ channel is calculated according to Shannon's theory as follows:

$$
C_{i, j}^{D}=X_{j} * B \log _{2}\left(1+\operatorname{SINR}_{i, j}^{D}\right) .
$$

\subsection{Problem formulation}

Our goal is to enhance QoS for VR users and D2D users and maximize multicast clusters throughput while the uplink delay of VR users is below a certain threshold. A binary matrix $\xi=\left[\zeta_{i}, j\right]_{I \times J}$ is defined to denote the situation that D2D clusters reuse VR user channels. $\zeta_{i, j}=1$ represents that $D_{j}$ reuse the channel of $\mathrm{VR}_{i}$. If not, $\zeta_{i, j}=0$. $P_{j}^{D, M A X}$ and $P_{i}^{V R, M A X}$ represent the maximum transmission power of $D_{j}$ and $\mathrm{VR}_{i}$, respectively. $\Gamma_{\mathrm{VR}, i}$ and $\Gamma_{D, j}$ represent the minimum SINR of $\mathrm{VR}_{i}$ and $D_{j}$, respectively. The optimization problem is expressed by a mathematical formula as follows:

$$
\begin{aligned}
& V_{i}=V_{i}^{P}\left(Z_{i}^{V R}\right)+V_{i}^{T} \leq \text { thord, } \\
& \underset{\zeta_{i, j}}{\operatorname{argmax}} \sum_{\mathrm{i}=1}^{\mathrm{I}} \sum_{\mathrm{j}=1}^{\mathrm{J}} c_{i, j}^{D}\left(P_{i, j}^{D}, P_{i, j}^{V R}\right), \\
& 0 \leq \sum_{\mathrm{i}=1}^{\mathrm{I}} \sum_{j=1}^{\mathrm{J}} \zeta_{i, j} \leq I, 0 \leq \sum_{i=1}^{J} \zeta_{i, j} \leq 1, \\
& \operatorname{SINR}_{i, j}^{V R}\left(P_{i, j}^{D}, P_{i, j}^{V R}\right) \geq \Gamma_{V R, i} \\
& \operatorname{SINR}_{i, j}^{D}\left(P_{i, j}^{D}, P_{i, j}^{V R}\right) \geq \Gamma_{D, j}, \\
& 0 \leq P_{i, j}^{V R} \leq P_{i}^{V R, M A X}, 0 \leq P_{i, j}^{D} \leq P_{j}^{D, M A X} .
\end{aligned}
$$

Equations (9) and (10) are our objective functions. Equation (9) guarantees that the uplink transmission delay of VR users is within the user's tolerable delay range, while throd denotes the threshold. Equation (10) is used to obtain the maximum throughput of D2D cluster. Constraint (11) is used to ensure that the channel resources of a VR user can only be reused by one D2D cluster, but a D2D cluster can multiplex multiple VR channels. Constraint (12) indicates that in the case of channel multiplexing, the QoS requirements of D2D users and VR users are met. Constraint (13) restricts the users' transmission power.

\section{Methods}

\subsection{Power control based on geometric programming}

To improve system throughput, it needs to allocate power between D2D clusters and VR users properly. In cellular networks, $D_{j}$ reuses $V R_{i}$ channel resources, and when both of them send information at the same time, there will be interference between them. Because the interference is related to power, by reducing the transmission power of $D_{j}$ and $V R_{i}$ the interference between $D_{j}$ and $V \mathrm{R}_{i}$ will be reduced. However, this will lead to the reduction of receiving power, SINR, and transmission power. To ensure the system's QoS, the optimal power of VR users and D2D clusters must be used. According to the SINR and power constraints of VR users and D2D clusters, the power optimization problem is expressed as follows:

$$
R=\left\{\begin{array}{c}
\left(P_{i, j}^{D *}, P_{i, j}^{V R *}\right)=\operatorname{argmax}\left(C_{i, j}^{D}\right),\left(P_{i, j}^{D}, P_{i, j}^{V R}\right) \in R, \\
\left(P_{i, j}^{D}, P_{i, j}^{V R}\right): 0 \leq P_{i, j}^{D} \leq P_{j}^{D, \operatorname{MAX}}, \\
0 \leq P_{i, j}^{V R} \leq P_{i}^{V R, \operatorname{MAX}}, \\
\operatorname{SINR}_{i, j}^{V R} \geq \Gamma_{V R, i}, \operatorname{SINR}_{i, j}^{D} \geq \Gamma_{D, j}, \\
V_{i}=V_{i}^{P}\left(Z_{i}^{V R}\right)+V_{i}^{T} \leq \text { thord }
\end{array}\right)
$$

In Eq. (14), both D2D clusters and VR users are constrained by maximum transmission power and minimum SINR, and $R$ is the feasible region. This problem is a convex optimization problem. We can find the optimal powers of D2D clusters and VR users in the feasible region. Linear programming is an effective method to solve this problem.

As shown in Fig. 2, $l_{\mathrm{vr}}$ and $l_{d}$ represent SINR of $\mathrm{VR}_{i}$ and $D_{j}$ that meet power limits. The right side of $l_{d}$ satisfies the limit of minimum SINR, while the upper side of $l_{\mathrm{vr}}$ satisfies the limit of minimum SINR. Hence, the shaded part in the figure is the feasible area, and the optimal power pair is within the area. According to Reference [24], the optimal power solution includes at least one maximum power in, i.e., $P_{i, j}^{V R}=P_{i}^{V R, \operatorname{MAX}}$ or $P_{i, j}^{D}=P_{j}^{D, M A X}$. Therefore, the optimal power solution is at the vertex of the feasible region. As shown in Fig. 2, the optimal power pair corresponds to 

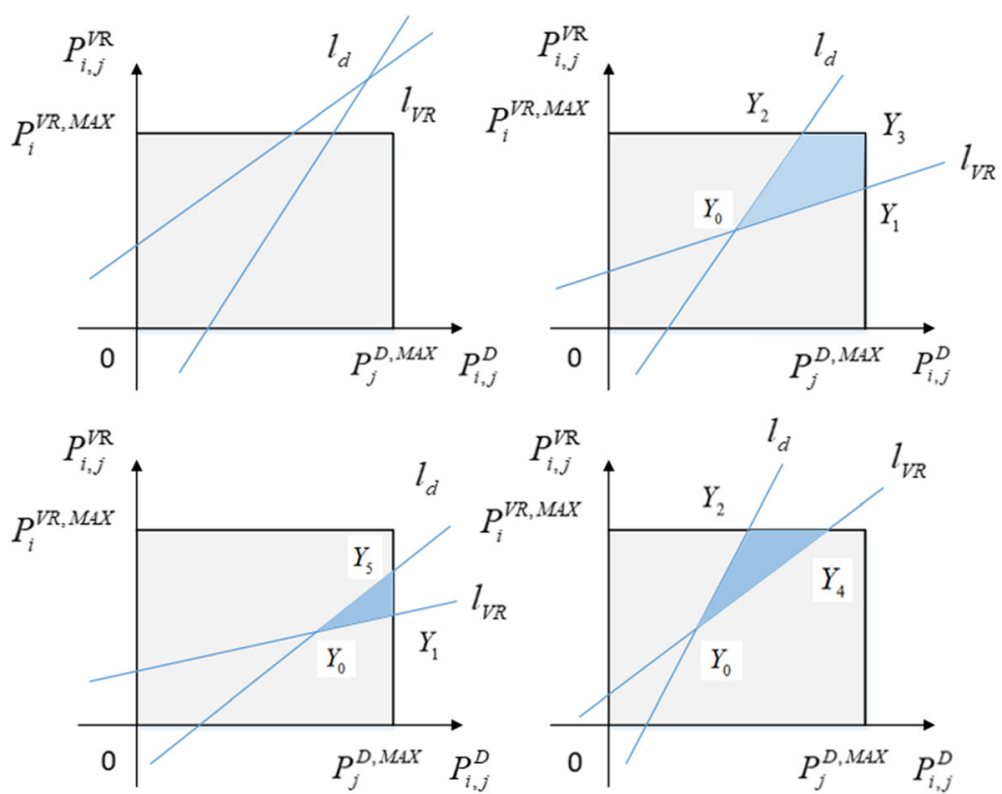

Fig. 2 Linear programming of $P_{i, j}^{D}$ and $P_{i, j}^{V R}$ power control. The figure shows the possible shape of the feasible region under different wireless channel conditions. $P_{j}^{D, M A X}$ and $P_{i}^{V R, M A X}$ denote the maximum transmission power of $D_{j}$ and $V R_{j}$ respectively. $I_{v r}$ and $l_{d}$ represent SINR constraints of transmission power for $D_{j}$ and $V R_{i}$

points $Y_{1}, Y_{2}, Y_{3}, Y_{4}$, and $Y_{5}$. In $Y_{1}\left(P_{j}^{D, M A X}, P_{i, j}^{V R, Y_{1}}\right), P_{i, j}^{V R, Y_{1}}$ is the intersection of $l_{\mathrm{vr}}$ and $P_{j}^{D, \operatorname{MAX}}$ when $\operatorname{SINR}_{i, j}^{V R}=\Gamma_{V R, j}$. In $Y_{2}\left(P_{i, j}^{D, Y_{2}}, P_{i}^{V R, \operatorname{MAX}}\right), P_{i}^{V R, \operatorname{MAX}}$ is the intersection of $l_{d}$ and $P_{i, j}^{V R, \operatorname{MAX}}$ when $\operatorname{SINR}_{i, j}^{D}=\Gamma_{D, j} \cdot Y_{3}$ is $\left(P_{j}^{D, \mathrm{MAX}}, P_{i}^{V R, \operatorname{MAX}}\right)$. In $Y_{4}\left(P_{i, j}^{D, Y_{4}}, P_{i}^{V R, \operatorname{MAX}}\right), P_{i, j}^{D, Y_{4}}$ is the intersection of $l_{\mathrm{vr}}$ and $P_{i, j}^{V R, \operatorname{MAX}}$ when $\operatorname{SINR}_{i, j}^{V R}=\Gamma_{V R, i}$. In $Y_{5}\left(P_{j}^{D, \mathrm{MAX}}, P_{i, j}^{V R, Y_{5}}\right)$, $P_{i, j}^{V R, Y_{5}}$ is the intersection of $l_{d}$ and $P_{j}^{D, \operatorname{MAX}}$ when $\operatorname{SINR}_{i, j}^{D}=\Gamma_{D, j}$. The results are as follows:

$$
\begin{aligned}
& P_{i, j}^{V R, Y_{1}}=\frac{\Gamma_{V R, i}\left(\sigma^{2}+P_{j}^{D, \operatorname{MAx}} h_{j, B}\right)}{h_{i, B}}, \\
& P_{i, j}^{D, Y_{2}}=\frac{\Gamma_{D, i}\left(\sigma^{2}+P_{i}^{V R, \operatorname{MAx}} h_{i, d_{j, x}^{r}}\right)}{h_{j, d_{j, x}^{r}}^{r}}, \\
& P_{i, j}^{D, Y_{4}}=\frac{P_{\mathrm{i}}^{V R, \operatorname{MAX}} h_{i, B}-\Gamma_{V R, i} \sigma^{2}}{\Gamma_{V R, i} h_{j, B}}, \\
& P_{i, j}^{V R, Y_{5}}=\frac{P_{j}^{D, \mathrm{MAX}} h_{i, d_{j, x}^{r}}-\Gamma_{D, j} \sigma^{2}}{\Gamma_{D, j} h_{i, d_{j, x}^{r}}} .
\end{aligned}
$$

By calculating the throughput of the above four optimal power pairs and judging whether the corresponding uplink transmission delay of VR users meets the threshold requirement, we compare the results to get the optimal power pair and the optimal throughput increment which finally meets the system requirements.

\subsection{Channel allocation based on Hungarian algorithm}

Based on the calculation for the optimal power pairs above, we can find out the optimal transmission power between $D_{j}$ of any D2D cluster and $V R_{i}$, and obtain the throughput between them. To find the optimal solution, we must search for all the mappings between D2D clusters and VR users [25]. This problem is transformed into the optimal matching problem, and the maximum weight binary matching algorithm is used to solve the optimal channel allocation.

As shown in Fig. 3, we construct a bipartite graph $G$, in which the set $S_{\mathrm{D}}$ and $S_{\mathrm{VR}}$ are two sets of vertices of $D_{j}$ and $V R_{i}$, respectively. If $V R_{i}$ is a candidate for $D_{j}$ to reuse channel resources, then there is a connection edge between two vertices, and the weight of the edge is the throughput of $D_{j}$; if not, the virtual edge weight between the two vertices is zero. In graph theory, the Hungarian algorithm is suitable for completely symmetric bipartite graphs. Therefore, we need to transform asymmetric bipartite graphs $G$ into symmetric bipartite graphs. As shown in Fig. 3, the specific method is as follows: (1) if $I$ is $\partial$ times of $J$, i.e., $I=\partial \times \mathrm{J}$, then adding $(\partial-1)$ vertices with original weighted edges to set $\mathrm{D}$ is to copy $(\partial-1)$ original clusters. 2$)$ If $\mathrm{I}=\partial \% \mathrm{I}+\mathrm{h}$ we add $\partial$ vertices with original weighted edges to the set $S_{\mathrm{D}}$ and $J-h$ virtual vertices with zero weights to the set $S_{\mathrm{VR}}$.

Firstly, the weights of the edges between vertex $S_{D}$ and $\mathrm{S}_{\mathrm{VR}}$ are calculated based on the optimal power pair between $D_{j}$ and $V R_{i}$. Then, we use Hungarian algorithm to find the 
best match between $D_{j}$ and $V R_{i}$. As shown in Fig. 3a, the weights of $V R_{1}$ are the largest in all channels of $D_{1}$ reusing and $V R_{5}$ is the largest in all channels of $D_{1}$ copy reusing, so the channels of $D_{1}$ reusing $V R_{1}$ and $V R_{5}$ are the best, that is, the channels of $D_{1}$ and $V R_{1}$ and $V R_{5}$ are the best matching.

\section{Experiments and results}

In this section, we will simulate and analyze the proposed scheme. In an area of $0.5 \mathrm{~km} \times 0.5 \mathrm{~km}$, an SBS is in the center, and VR users and D2D multicast clusters are randomly distributed. Each D2D cluster is a circular area with a radius of $0.1 \mathrm{~km}$ by fault. The cluster head locates in the center of the cluster. Before resource allocation, we assume that all users share average bandwidth and achieve the lowest QoS. The transmission rate of wireless virtual reality is required to be $25.32 \mathrm{Mbit} / \mathrm{s}$. The maximum transmission power of VR users and SBS is $21 \mathrm{dbm}$ and $42 \mathrm{dbm}$, respectively. The specific parameter settings are shown in Table 2 below.

The objective of our proposed method is to optimize the QoS of VR video content sharing using D2D technology on the premise that the uplink transmission delay of VR is less than the specified threshold. The mainstream view in the industry is that when using immersive terminals. The latency from the user sending the request to receiving the content cannot exceed $20 \mathrm{~ms}$, if not, it will cause vertigo. For this reason, we set the threshold of VR uplink transmission delay to $10 \mathrm{~ms}$ by default. Since power allocation and channel allocation are mainly to improve the overall throughput performance, we use throughput as an evaluation index. Firstly, we analyze the impact of the number of D2D cluster users on the throughput of D2D cluster and VR user before and after channel resource reuse under the condition that the upstream delay of VR is $10 \mathrm{~ms}, 15$ $\mathrm{ms}$, and $20 \mathrm{~ms}$, respectively. Secondly, the influence of the radius change of the D2D cluster is also analyzed. Then, we compare the proposed allocation scheme with heuristic and stochastic schemes to evaluate their performance. Besides, we analyze the changes of video resolution in D2D cluster users before and after channels reusing.

Figure 4 shows how the throughput of D2D clusters varies as the number of users in the D2D clusters changes. As the increase of users in D2D clusters, the throughput of all D2D clusters increases continuously. The throughput of

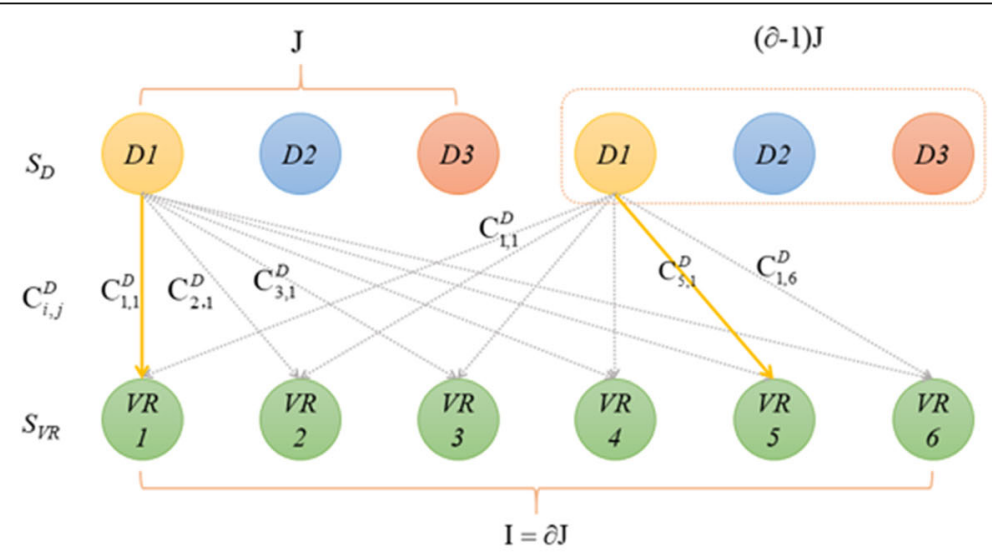

(a)

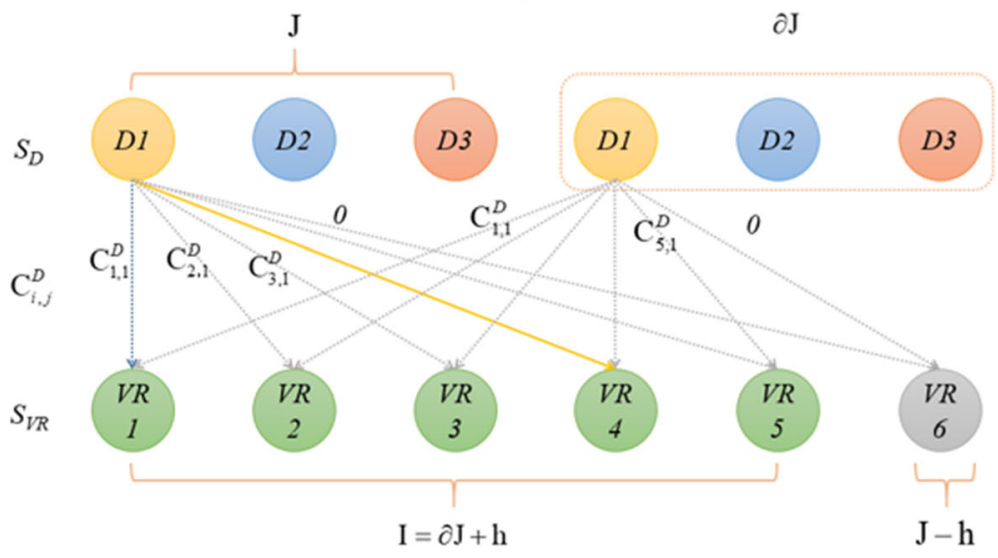

(b)

Fig. 3 Bipartite matching of $D_{j}$ multiplexed $V R_{i}$ channels. a Matching in the case of $I=\partial \times J$. b Matching in the case of $I=\partial \times J+h$ 
Table 2 Parameters of simulation

\begin{tabular}{ll}
\hline Parameter & Value \\
\hline SBS radius & $0.5 \mathrm{~km}$ \\
D2D cluster radius & $0.1 \mathrm{~km}$ \\
Maximum transmission power & $21 \mathrm{dbm}$ \\
Uplink bandwidth & $10 \mathrm{MHz}$ \\
Noise spectral density & $-174 \mathrm{dBm} / \mathrm{Hz}$ \\
Path loss model for VR links & $128.1+37.6 \log (\mathrm{d}[\mathrm{km}])$ \\
Path loss model for D2D links & $148+40 \log (\mathrm{d}[\mathrm{km}])$ \\
$\Gamma_{V R, i} \Gamma_{D, j}$ & $3 \mathrm{~dB}$ \\
$\mathrm{~A}$ & $100 \mathrm{~kb}$ \\
$V$ & $500 \mathrm{~kb}$ \\
\hline
\end{tabular}

D2D clusters after reusing channels is higher than that of not reusing channels, and more users, the greater the throughput. And the more users, the greater the throughput increment. However, when there are more than 40 users in each D2D cluster, the increase of throughput becomes slow. This is owing to the increase of the number of users, which leads to the increase of interference between users in the cluster, resulting in the decrease of throughput increment. In addition, when the VR uplink delay is gradually increased by $10 \mathrm{~ms}, 15 \mathrm{~ms}$, and $20 \mathrm{~ms}$, respectively, we find that the D2D clusters throughput gradually increases. As the VR uplink delay increases, the transmission power of the VR users decreases, which lead to the interference of the VR users to the D2D clusters decreases.

Figure 5 illustrates the impact of changing the number of users in a D2D cluster on the throughput of virtual reality users. In Fig. 5, the throughput of VR users does not increase or decrease significantly as the increase of users in D2D clusters. The fluctuation of the curve is caused by the random generation of users' locations. The change of distance leads to the fluctuation of throughput, but the increment of VR throughput remains almost unchanged. The reason is that although the number of users in the D2D cluster increases, the radius of the D2D cluster does not change, which will not affect the worst channel quality. The effects of D2D cluster heads and SBS on the channel quality of VR are almost invariant. Through the analysis of Figs. 4 and 5, we find that the throughput gain of D2D clusters after reusing channel is significantly greater than the throughput reduction after VR users is reused. While guaranteeing the VR user experience, the system throughput is significantly improved by reusing channels. In addition, the VR uplink delay gradually increases, causing the transmission power of VR users to decrease. As a result, the uplink throughput of virtual reality users decreases.

Figure 6 shows that the total throughput of D2D clusters decreases with the increase of D2D radius. The reason is the increase of cluster radius, the coverage of D2D cluster becomes larger. When the number of users in each cluster is fixed, the range of random distribution of users becomes larger, and the channel quality between D2D cluster heads and intra-cluster users becomes worse. The rate of both D2D multicast depends on the worst channel quality, so the throughput of D2D cluster decreases. With the change of each D2D cluster radius, we also find that the throughput curve of D2D cluster decreases with the increase of VR upstream delay. However, the throughput of D2D clusters reusing VR channels resources is still better than that of non-multiplexing channels.

Figure 7 illustrates how the throughput of VR users varies as the D2D clusters radius change. In Fig. 7, the total throughput of VR users decreases with the increase of D2D clusters radius. As the cluster radius increases, the coverage

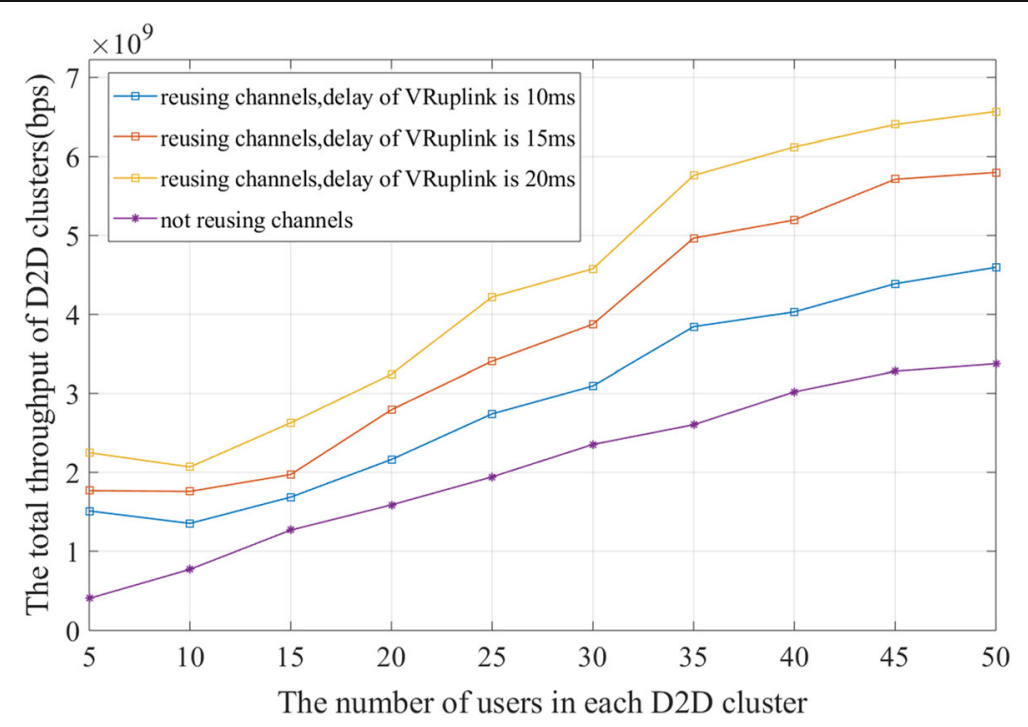

Fig. 4 The effect of changing the number of users in the D2D clusters on the total throughput of the D2D clusters 


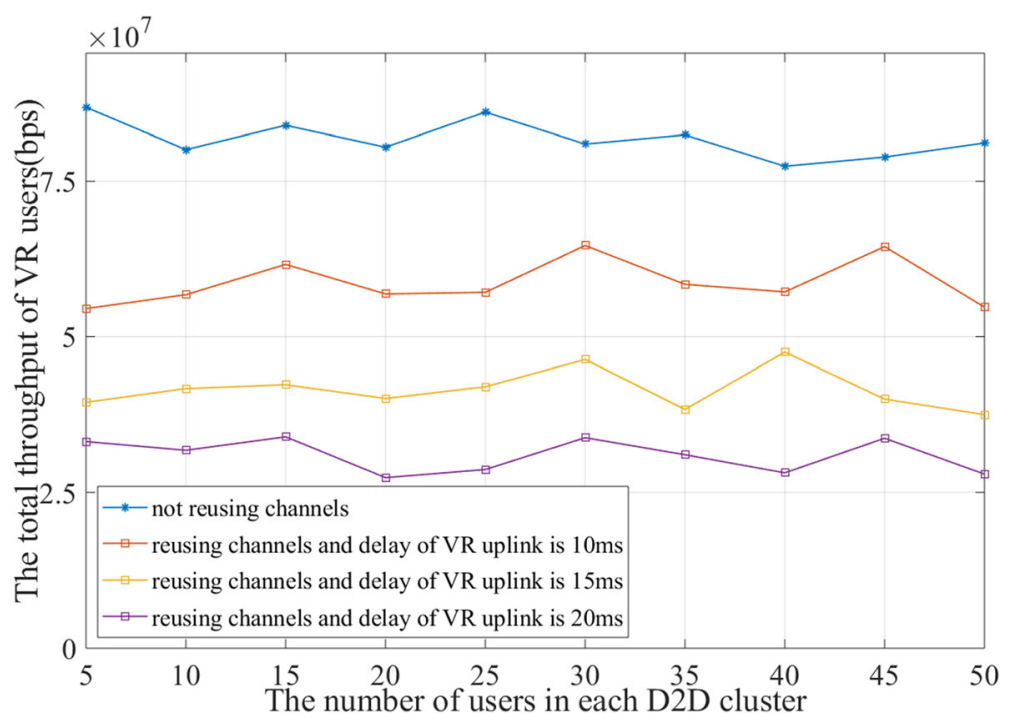

Fig. 5 The impact of the number of users in the D2D clusters on the total throughput of VR users

area of D2D communication increases, and the interference caused by D2D cluster reusing VR users' channel resource increases, which makes the total throughput of VR reduces. When the VR uplink delay is $10 \mathrm{~ms}$, the throughput is higher than that of the other two delays. The delay of $10 \mathrm{~ms}$ makes the user rarely feel dizzy during the immersive experience, and the corresponding throughput guarantees the quality of VR video transmission. When the VR users channels are not reused, the throughput of VR users is not interfered by the D2D clusters but only related to the channel gain between SBS and VR users. Although the locations of VR users are randomly allocated, the distance between VR users and SBS varies in a limited range. So the channel gain is almost unchanged, and the throughput of VR user channels is almost unchanged when they are not reused.
In Fig. 8, we compare the resource allocation scheme of this paper with heuristic and stochastic schemes. For the heuristic scheme, each D2D cluster searches for the best object in order. For the stochastic scheme, as long as Formula (14) is satisfied, we can get the cluster's reusable random resource allocation matrix. As shown in Fig. 8, the throughput of the three allocation schemes is better than that of the D2D clusters not reusing VR channels. Because the stochastic scheme cannot guarantee the quality of the allocated channels. Compared with the other two schemes, the throughput increment is smaller. When the number of users in each D2D cluster is 30 , the throughput of the proposed scheme is $50 \%$ and $12 \%$ higher than that of stochastic algorithm and heuristic algorithm, respectively. Because the proposed algorithm considers all possible channel allocation situations, and selects the one that makes the system

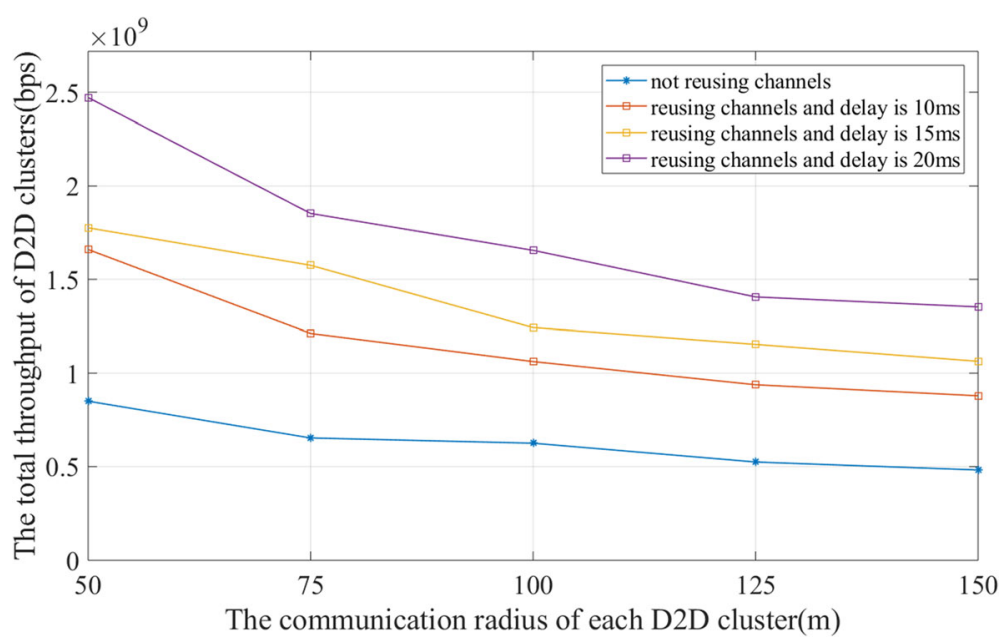

Fig. 6 The influence of the radius size of D2D clusters on the total throughput of D2D cluster 


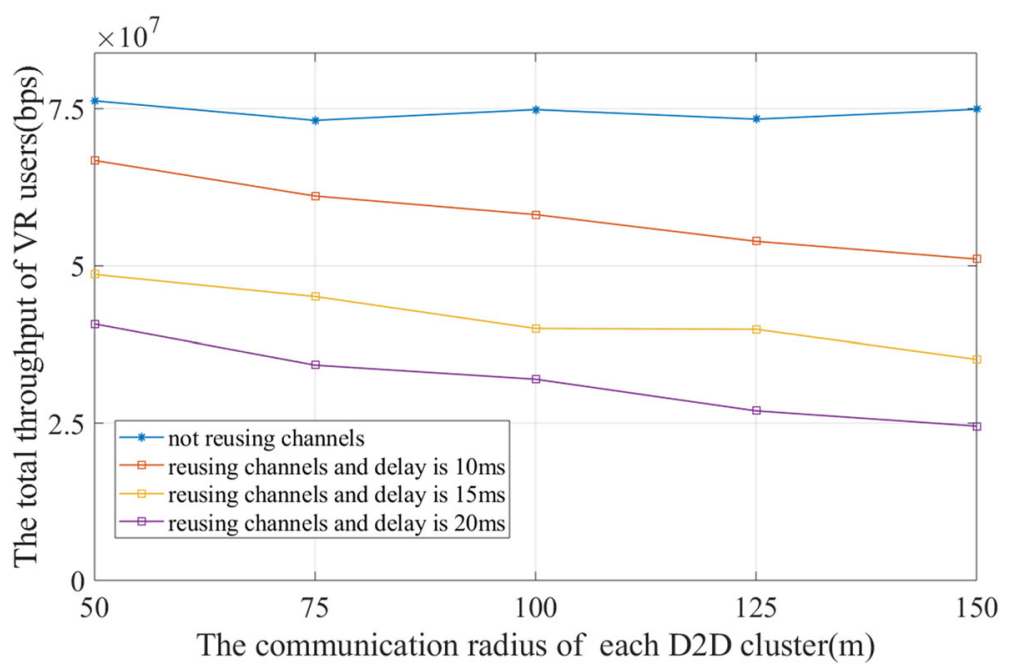

Fig. 7 The influence of the radius size of D2D clusters on the total throughput of VR users

performance the best, and the stochastic algorithm selects one from the set of multiplexing channels that meet the constraints, and the heuristic algorithm pursues the maximum benefit of a single D2D cluster, without considering the global optimization. The proposed scheme is superior to the heuristic scheme and stochastic scheme in terms of total system throughput.

In Fig. 9, we evaluate the video quality of D2D clusters for VR videos content sharing. With the development of VR, the level of software and hardware related to VR is also promoting. The resolution of the terminal screen has reached $4 \mathrm{~K}$ and the resolution of the full view is $8 \mathrm{~K}$. If the resolution is $4 \mathrm{~K}$, the network bandwidth requirement is 25 Mbps-100 Mbps. If the resolution is $8 \mathrm{~K}$, the network bandwidth requirement exceeds 100 Mbps. Based on the bandwidth requirements of the above resolution, we analyze the proportion of the resolution of the users in the D2D clusters as the D2D clusters radius change. We also set up 6 VR users, 12 D2D clusters, and 5 users per cluster. In the case where the number of users in the cluster is fixed and randomly distributed, as shown in Fig. 9, different colors indicate the proportion of users with different resolutions before and after the channels are reused. We found that increasing the D2D cluster radius from $50 \mathrm{~m}$ to $150 \mathrm{~m}$ leads to the decrease of clusters throughput, which results in the decrease of resolution. However, after D2D clusters reusing channels, the resolution is improved obviously, and the proportion of the video resolution of $8 \mathrm{~K}$ is increased.

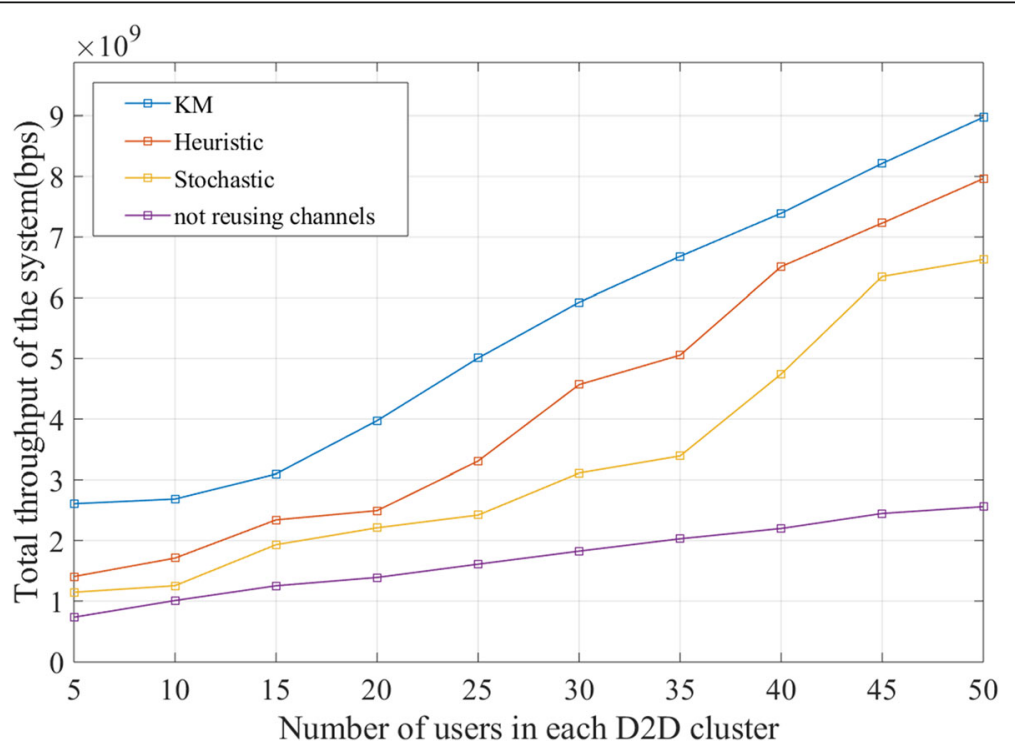

Fig. 8 Throughput comparison of resource allocation schemes 


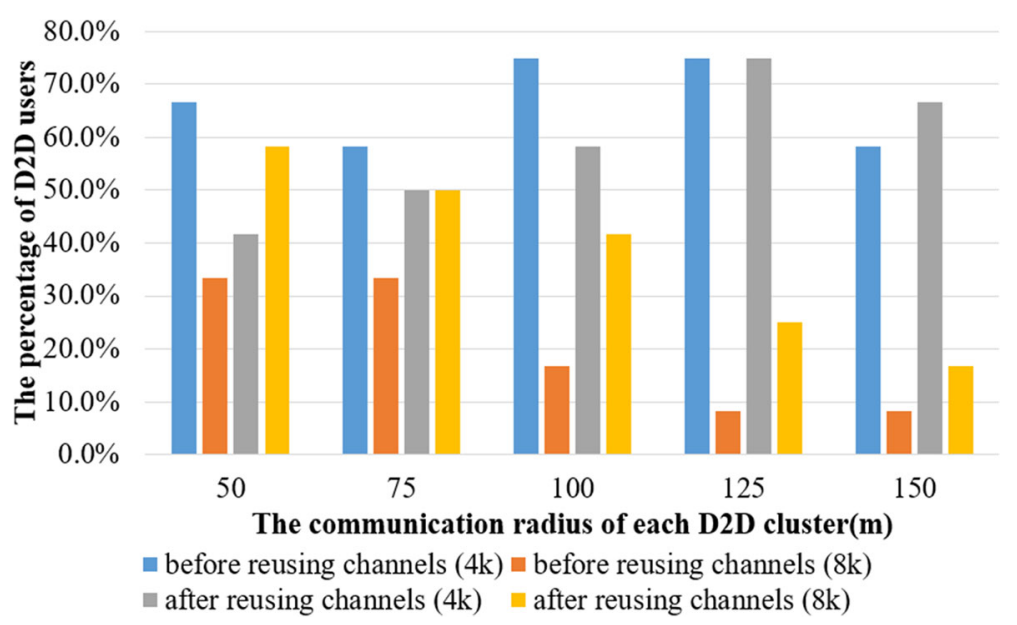

Fig. 9 The influence of D2D communication radius on the revolution of VR videos

The reason is that the user throughput increases after the channel reusing, which can support higher resolution. Thus, the proposed mechanism improves the VR video resolution and improves the user experience.

Through the analysis of the above simulation diagrams, we find that our proposed scheme of content distribution can greatly improve the system throughput and guarantee the QoE of VR users and video quality. Our proposed resource allocation scheme is also significantly better than the other two schemes in throughput.

\section{Conclusions}

In this paper, we propose a scheme for wireless VR content sharing based on D2D multicasting technology. There are two VR communication modes in this scheme: VR users with the same VR content sharing intent constitute the D2D multicasting clusters, while other ones communicate directly with the base station. The D2D multicasting clusters reuse the uplink channel resources occupied by non-clustered VR users for communication. Then, we research the resource allocation of this scheme. We propose that the resource allocation optimization problem is to maximize the throughput of D2D multicasting clusters for video content sharing and to guarantee the quality of VR services. For this reason, we consider key VR performance such as uplink transmission latency and tracking processing latency. We put forward a two-step algorithm to solve the above optimization problem. The optimal power pair between the D2D clusters and VR users is obtained by linear programming. Then, the problem of reusing channel is transformed into a bipartite graph, and the Hungarian algorithm is used to achieve the optimal channel allocation. Finally, the performance of the proposed scheme is evaluated by simulation. The numerical simulations verify the proposed scheme can effectively improve the throughput for VR video content sharing and the clarity of VR content transmission.

\section{Abbreviations}

VR: Virtual reality; QoS: Quality of service; D2D: Device-to-device; QoE: Quality of experience; mmWave: Millimeter wave; CMT-DA: Concurrence multipath transmission-distortion aware; ESN: Echo state network; SBS: Small base station; CPU: Central processing unit; MTP: Motion-to-photons latency; SINR: Signal-to-noise ratio

\section{Acknowledgements}

Not applicable.

\begin{abstract}
Authors' contributions
Yang Yang was in charge of the major theoretical analysis, algorithm design, experimental simulation, and wrote the manuscript. Lei Feng put forward the idea of this paper. Other authors were in charge of part of the theoretical analysis and experiment. All authors read and approved the final manuscript.
\end{abstract}

\section{Funding}

This work is partly supported by the Beijing Natural Science Fund-Haidian Original Innovation Joint Fund (L192003), the Fundamental Research Funds for the Central Universities under grant 2019RC09 and State Grid Science and Technology project "Analysis of Power Wireless Private Network Evolution and 4G/5G Technology Application" (grant no. .5700-201941235A-0-0-00).

\section{Competing interests}

The authors declare that they have no competing interests.

\section{Author details}

${ }^{1}$ Institute of Network Technology, Beijing University of Posts and Telecommunications, Xitucheng Road, Beijing 100876, China. ${ }^{2}$ Department of Computer Science and Communications Engineering, Waseda University, Tokyo 169-0072, Japan. ${ }^{3}$ Beijing Fibrlink Communications Co., Ltd., Beijing, China.

Received: 1 November 2019 Accepted: 13 March 2020 Published online: 03 June 2020

\section{References}

1. Y. Wen, X. Zhu, J.J.P.C. Rodrigues, W.C. Chang, Cloud mobile media: reflections and outlook. IEEE Transactions on Multimedia 16(4), 885-902 (2014)

2. P. Rosedale, Virtual reality: The next disruptor: A new kind of worldwide communication. IEEE Consumer Electronics Magazine 6(1), 48-50 (2017)

3. Spolitis, S., Bobrovs, V., Ivanovs, G.: Latency causes and reduction in optical metro networks (invited). In: Spie Photonics West Opto (2013) 
4. Lo W.C., Fan C.L., Yen S.C., Hsu C.H.: Performance measurements of 360 video streaming to head-mounted displays over live $4 \mathrm{~g}$ cellular networks. In: Network Operations Management Symposium (2017)

5. G. Fodor, E. Dahlman, G. Mildh, S. Parkvall, N. Reider, G. Miklos, Z. Turanyi, Design aspects of network assisted device-to-device communications. Communications Magazine IEEE 50(3), 170-177 (2012)

6. Li, W., Tang, H., Wu, H., Stuber, G.L.: Resource allocation for d2d communications underlay in rayleigh fading channels. IEEE Trans. Veh. Technol. PP(99), 1-1 (2017)

7. A. Rovira, M. Slater, Reinforcement learning as a tool to make people move to a specific location in immersive virtual reality. Int. J. Hum. Comput. Stud. 98, 89-94 (2017)

8. M. Chen, W. Saad, C. Yin, "Liquid State Based Transfer Learning for $360^{\circ}$ Image Transmission in Wireless VR Networks," ICC 2019 - 2019 (IEEE International Conference on Communications (ICC), Shanghai, 2019), pp. 1-6

9. Y. Hu, S. Xie, Y. Xu and J. Sun, "Dynamic VR live streaming over MMT," 2017 IEEE International Symposium on Broadband Multimedia Systems and Broadcasting (BMSB), Cagliari, 2017, pp. 1-4.

10. J. Wu, C. Bo, C. Yuen, Y. Shang, J. Chen, Distortion-aware concurrent multipath transfer for mobile video streaming in heterogeneous wireless networks. IEEE Trans. Mob. Comput. 14(4), 688-701 (2014)

11. J. Wu, C. Yuen, N.M. Cheung, J. Chen, C.W. Chen, Modeling and optimization of high frame rate video transmission over wireless networks. IEEE Trans, Wirel. Commun. 15(4), 2713-2726 (2016)

12. Y. Liu, Y. Liu and H. Yang, "A progressive transmission scheme for 3D models in VR/AR based on UEP-LT code," 2017 IEEE 28th Annual International Symposium on Personal, Indoor, and Mobile Radio Communications (PIMRC), Montreal, QC, 2017, pp. 1-6.

13. Chen, M., Saad, W., Yin, C.: Virtual reality over wireless networks: Quality-ofservice model and learning-based resource management. IEEE Trans. Commun. PP(99), 1-1 (2017)

14. E. Bastug, M. Bennis, M. Medard, M. Debbah, Toward interconnected virtual reality: opportunities, challenges, and enablers. IEEE Commun. Mag. 55(6), 110-117 (2017)

15. Militano, L., Condoluci, M., Araniti, G., Molinaro, A., lera, A., Fitzek, F.H.P.: Wi-fi cooperation or d2d-based multicast content distribution in Ite-a: A comparative analysis. In: IEEE International Conference on Communications Workshops (2014)

16. F. Lei, Z. Pan, F. Zhou, M. Yin, Y. Peng, W. Li, X. Qiu, Resource allocation for $5 \mathrm{~g}$ d2d multicast content sharing in social-aware cellular networks. IEEE Commun. Mag. 56(3), 112-118 (2018)

17. Lei, W., Gao, L., Zhang, A., Chen, M.: Social-aware file-sharing mechanism for device-to-device communications. In: International Conference on Wireless Communications \& Signal Processing (2015)

18. Bo, P., Hu, C., Tao, P., Yang, Y., Wang, W.: A resource allocation scheme for $\mathrm{d} 2 \mathrm{~d}$ multicast with qos protection in ofdma-based systems. In: IEEE International Symposium on Personal Indoor \& Mobile Radio Communications (2013)

19. Yang, L., Dan, W., Xu, S., Cai, Y.: Social-aware energy-efficient joint mode selection and link allocation in d2d communications. In: International Conference on Wireless Communications \& Signal Processing (2016)

20. C. Yang, J. Tao, C. Xu, J. Zhang, Social-aware video multicast based on deviceto-device communications. IEEE Trans. Mob. Comput. 15(6), 1528-1539 (2016)

21. A. Mehrabi, et al., "D2D-enabled collaborative edge caching and processing with adaptive mobile video streaming," in 2019 IEEE 20th International Symposium on "A World of Wireless, Mobile and Multimedia Networks" (WoWMoM), pp. 1-10, June 2019

22. H. Huang, B. Liu, L. Chen, W. Xiang, M. Hu, Y. Tao, D2d-assisted vr video precaching strategy. IEEE Access 6, 61886-61895 (2018)

23. L. Wei, J. Cai, C. Foh, B. He, Qos-aware resource allocation for video transcoding in clouds. IEEE Transactions on Circuits Systems for Video Technology 27(1), 49-61 (2017)

24. Pan, Z., Lei, F., Peng, Y., Li, W., Qiu, X.: A social-aware resource allocation for $5 \mathrm{~g}$ device-to-device multicast communication. IEEE Access PP(99), 1-1 (2017)

25. Bhardwaj, A., Agnihotri, S.: A resource allocation scheme for device-to-device multicast in cellular networks. In: IEEE International Symposium on Personal (2015)

\section{Publisher's Note}

Springer Nature remains neutral with regard to jurisdictional claims in published maps and institutional affiliations.

\section{Submit your manuscript to a SpringerOpen ${ }^{\circ}$ journal and benefit from:}

- Convenient online submission

- Rigorous peer review

- Open access: articles freely available online

- High visibility within the field

- Retaining the copyright to your article

Submit your next manuscript at $\boldsymbol{\nabla}$ springeropen.com 